\title{
The Enlightenments and Perplexities of Research on Legal Pluralism Theory in China
}

\author{
Qu Yanhong \\ Inner Mongolia University, Saihan district, Hohhot, Inner Mongolia, China \\ Mudanjiang Normal University, Aimin district, Mudanjiang, Heilongjiang, China \\ Email:july7911@126.com \\ ${ }^{*}$ Corresponding author: Qu Yanhong
}

Keywords: Legal pluralism, Enlightenment, Perplexity, China.

\begin{abstract}
Legal pluralism theory is the core proposition of social science research on law. Different disciplines have demonstrated legal pluralism with their unique disciplinary perspectives and research methods. In general, although legal pluralism still has some theoretical dilemmas, the empirical research method of the social science path (anthropology and sociology) and its basic conclusion "legal pluralism refers to the interaction between the non-national law system and the national law system" have the significance of breaking the barriers of discipline and exploring the substantive rationality of law. Nowadays, Chinese society is at a stage where traditional ritual-law has been or is being lost, while the modern rule of law has not yet been established. The theory of legal pluralism has considerable enlightening effect for understanding and treating correctly the multiple social control methods and their symbiotic interaction with national law in China, however, the current research on legal pluralism also faces some perplexities.
\end{abstract}

\section{中国法律多元理论研究的启示及困惑 \\ 曲艳红 \\ 内蒙古大学, 赛罕区, 呼和浩特, 内蒙古, 中国 \\ 牡丹江师范学院, 爱民区, 牡丹江, 黑龙江, 中国 \\ Email:july7911@126.com \\ *通讯作者: 曲艳红}

关键词：法律多元；启示；困惑；中国

中文摘要. 法律多元理论是法的社会科学研究的核心命题。不同学科以其特有的学科视角和研 究方法, 对法律多元展开了论证。综合看来, 尽管法律多元论仍存在一些理论困境, 但社会 科学路径 (指人类学与社会学) 的经验研究方法及其基本结论 “法律多元指非国家法体系与 国家法体系的互动”, 对于法学研究具有打破学科藩篱、探寻法的实质理性的意义。当今中国 社会处于传统礼法已经或正在消失、现代法治尚未建立的阶段, 法律多元理论对于认识并正 确对待作用于社会秩序的多种社会控制手段及其与国家法共生互动的模式, 具有相当的启示 作用，同时现在的法律多元研究也面临着一些待解的困惑。

\section{1. 引言}

法律多元理论是西方法律人类学与法社会学的核心概念，其形成于西方殖民时期，被人 类学家用于描绘殖民地地区 “本土法”与 “西方移植法” 的竞争、共存状态。后随着时代的 发展及学者研究的深入, 从20世纪70年代开始, 法律多元理论也被用来解释西方社会主权国 家中 “非国家法”与 “国家法” 的博弯状态及对国家法的规避行为。时至今日, “法律多元” 
除了在上述语境下使用, 中外学者们也将研究触角延伸到全球化过程中出现的人员的跨国移 动和文化的交流、传播所导致的法律多元状况, 从研究传统来说, 是法人类学、法社会学学 者开辟和引领了法律多元的思潮, 并最终进入法学理论界视野, 产生了各相关学科之间的对 话与关于 “什么是法”、“非国家法定位”、“非国家法与国家法关系”等问题的论辩。

\section{2. 关于法律多元理论的中西典型作者及观点}

法律多元理论产生于上世纪中叶西方人类学家的研究中, 西方学者构筑了该理论的基本 框架, 在上世纪末, 中国学者也开始用法律多元理论来解释官方法与民间法、国家制定法与 少数民族习惯法共存的状态。

\section{1 国外代表性学者及贡献}

西方国家对法律多元理论的研究时间较长、程度较深、范围较广。有致力于关注由异质 法 (如国家法、国际法、宗教法、习惯法等) 之间互动引起的理论及实践问题的专门委员会 “The Commission on Legal Pluralism (CLP) (原名为The Commission on Folk Law and Legal Pluralism)”，该委员会于1978年由The International Union of Anthropological and Ethnological Sciences (IUAES)建立在新西兰奈梅亨大学 (Nijmegen University), 机构有专门出版物Journal of Legal Pluralism and Unofficial Law。西方代表性学者的著作及主要贡献如下：（1） M.B. Hooker, "Legal Pluralism: An Introduction to Colonial and Neo-Colonial Law", Oxford, Clarendon Press, 1975. 胡克以殖民统治为背景, 对法律多元进行了分析, 自此 “法律多元才开始出现在 世界学术界的前沿” (千叶正士语)；（2）J.Griffiths, “What Is Legal Pluralism”, Journal of Legal Pluralism and Unofficial Law, nr.24, 1986. 格里菲斯对 “法律多元” 及其相关概念进行了细致 分析, 其观点及论述得到了众多学者的承认和引用; (3) B.de Sousa Santos, “Law: A Map of Misreading___Toward a Postmodern Conception of Law”, Journal of Law and Society, 14, 1987. 桑托斯是将 “法律多元” 置于 “后现代法学”、“全球化” 背景下讨论的典型学者; (4) Sally Engle Merry, “Legal Pluralism”, Law and Society Review, Vol.22, Number5, 1988. 梅丽在将法律多元主 义划分为 “经典的法律多元主义” (Classic Legal Pluralism)和 “新法律多元主义” (New Legal Pluralism)的基础上，分析了 “国家法” 与 “非国家法”之间的互动、博弯关系; (5) 千叶正 士, “法律多元一从日本法律文化迈向一般理论”, 中国政法大学出版社, 1997。千叶正士 为站在非西方立场、从本民族经验出发对法律多元进行理论建构的东方学者先驱, 其将法律 的多元解析为法律的三重二分结构; (6)B. Tamanaha, “The Folly of the Social Scientific Concept of Legal Pluralism”, 20 Journal of Law and Society, 1993.及B. Tamanaha, “A Non-Essentialist Version of Legal Pluralism”, 27 Journal of Law and Society, 2000.塔玛纳哈是参与到 “法律多元” 讨论的法学理论家代表人物, 其拒绝为法律确定普适性的概念, 而主张在具体社会场景中确 认法律, 在 “非本质主义法律观” 下理解法律多元; (7) W. Twining, “Normative and Legal Pluralism: A Global Perspective”, 20 Duke Journal of Comparative and International Law, 2010. 退宁与塔玛纳哈观点相近, 并指出将法律多元理解为 “规范多元” 的思路。

\section{2 中国代表性研究}

中国社会未曾经历过彻底的殖民统治, 但中国自古多民族, 更出现过两个少数民族建立 的封建王朝元和清, 因而中国的法律制度与法律文化在新中国建立前一直是 “多元一体” 的。 [1]中国学者对法律多元理论研究开始于二十世纪末, 其主要关涉以下几个领域: (1) “官方法” 与 “民间法”、“少数民族习惯法”与 “国家制定法” 的关系及整合; (2) 法治建设中对 “本 土资源” 的合理利用;（3）对中国古代 “国家法”与 “习惯法” 的搜集与研究; (4) 法律多 元基本问题探析及全球化背景下的法律多元理论, 等等。代表性学者及论著包括 (但不限于):

第一方面：谢晖，论当代中国官方与民间的法律沟通，学习与探索，2000（01）; 张晓辉， 法文化探论主持者言, 思想战线, 2004（6); 官波, 少数民族习惯法与国家法的依存和冲突 
少数民族地区法律多元个案透视，思想战线，2004（6); 刘建刚, 法律多元视野下的村 规民约实证研究——贵州省雷山县西江千户苗寨为例, 中央民族大学博士学位论文, 2013。 第二方面: 苏力, 法律规避和法律多元, 中外法学, 1993（6)、文化多元与法律多元: 人类 学研究对法学研究的启发, 载周星、王铭铭执行主编, 社会文化人类学讲演集, 天津人民出 版社, 1997; 赵旭东, 权利与公正—乡土社会的纠纷解决与权威多元, 天津古籍出版社, 2003。第三方面: 梁治平, 清代习惯法: 社会与国家, 中国政法大学出版社, 1996、中国法 律史上的民间法一一兼论中国古代法律的多元格局, 中国文化, 1997 (Z1); 王志强, 法律多 元视角下的清代国家法, 北京大学出版社, 2003; 白京兰, 一体与多元: 清代新疆法律研究 (1759-1911年), 中国政法大学博士学位论文, 2011。第四方面: 李理, 千叶正士的法律多 元观及中国的法律多元问题, 贵州师范大学学报 (社会科学版), 2005 (2); 张钧, 法律多元 理论及其在中国的新发展, 法学评论, 2010（4); 张德沝, 法律多元主义及其中国语境: 规 范多元化, 政法论丛, 2013（5); 杨静哲, 法律多元论: 轨迹、困境与出路, 法律科学（西 北政法大学学报), 2013 (2); 徐爱国, 法理念的文化冲突与中国法律的多元属性, 社会科学 研究, 2014 (6); 周晓虹, 透视全球化背景下的法律多元论, 吉林大学博士学位论文, 2006。

概言之, 中国的法律多元研究在法人类学、法社会学、法律史学、法理学等均有涉及并 渐成气候, 在法律多元理论的观照下, 认真观察并审慎对待社会中存在的道德、宗教、少数 民族习惯法、乡规民约、组织章程等多元社会规范, 对中国的现代法治社会建设具有重要的 指导意义。

\section{3. 法律多元理论探究}

“法律多元” 理论脱胎于法人类学对殖民地、后殖民地 “法秩序” 的观察和研究, 后来 法社会学、法理学参与到讨论中, 不同学科采用了不同的研究路径, 同时也丰富了理论的内 涵与外延。

\section{1 “法律多元” 的理论源头}

总的来说是法的社会科学研究主导了这一理论的产生和发展。18、19世纪的法典化运动 的抵制者一一萨维尼（F. C. V. Savigny）对法的 “民族精神” 的图腾式的崇拜和埃利希（E. Ehrlich）对 “活法” 的发掘, 被视为这一传统（指法律多元：笔者注）的奠基者。[2]在笔者 看来, 指出法与国体政体、地理环境、宗教风俗等方面联系的 “法的精神” 的孟德斯鸭传世 之作《论法的精神》, 及至二十世纪的人类学代表人物马林诺夫斯基与霍贝尔对土著部落形态 各异的 “法” 的描述、阐释人类学家格尔茨作出的界定 “法为地方性知识” 等众多经典论断, 都暗含着 “法律多元” 之精神, 即各时代、各国家、各地区的法律（规则）绝非一种形式、 一个体系。晚近一般法理学的代表人物塔玛纳哈（Tamanaha）及退宁（Twining）, 不再直于 对法的本质的探究、主张连通法律与社会、“不排斥借鉴社会科学的洞见”，[3]使得对于 “法 律多元” 的研究不仅进一步拓展到主权国家社会中 “非国家法”与 “国家法” 并存的 “二元” 面向, 而且社会学的经验研究与法学的规范研究的结合, 使法律多元理论更好地规避了两个 学科在该方面研究的不足, 理论更为丰富与完善。

\section{2 “法律多元” 的含义辨析}

任何一个理论和思潮产生之后, 研究者们都会展开对基本概念的剖析, 法律多元理论的 研究也不例外。但对同一概念, 不同学科从其特有的研究视角和研究惯式出发, 可能得出内 涵、外延不同的结论。

胡克（M.B.Hooker）在其著作《法律多元》中以殖民统治为社会背景, 指出 “同一情形 下有两个以上的法律相互作用的情况” 为法律多元。 ${ }^{[4]}$ 范德林登（Vanderlinden）认为社会中 由两种以上的法律秩序构成的规制性秩序称为 “法律多元”, 即在同一情形下有多种法律适用 
的状况。 ${ }^{[5]}$ 梅丽（Merry）从宏观视角指出, 同一社会共存多种法律制度的状况为法律多元。 [6]可见以上三位学者尽管侧重点不同, 但都是从社会学实证分析的角度切入, 界定法律多元 的本质是同一时空内共存多种法律制度。内德（Nader）认为社会群体的多样性必然导致法律 结构的多样性，并指出这些结构之间的关系 “相互独立、相互依赖、相互渗透或者三者共存”。 [7]格里菲斯（Griffiths）指出了法律多元是法的社会科学研究的新范式, 他认为社会法律秩序 的形成，不单独由国家决定，其来源于多个社会层面，并能够自我管理。[8]这两位学者都注 意到了引起法律多元的原因是社会结构/层面的多元, 并指出了多元的法律结构/秩序的能动 性。桑托斯（Santos）提出 “居间法制” (inter-legality) 的概念，指出由非正式、非官方的 修辞性法律所形成的法制与国家官方法律体系构成的法律秩序多元性为法律多元。[9]千叶正 士对法律的多元结构进行了剖析，其将法律解析为三层一一相对的对立结构，即 “官方法对 非官方法、法律规则对法律原理、固有法对移植法”，被学者称为 “法律的三重二分法”。[10] 以上两位学者都对 “法律多元” 进行了解构, 使法律多元的内容更为明确而层次化, 尤其千 叶正士的剖析使得法律多元理论内容具体丰满, 使后来的学者关注到了具体的二元对立情形。

中国学者对 “法律多元” 的界定，可以从以下角度切入：一为将中国古代朝代更替、地 域广博、民族众多背景下的法制历史描述为 “朝廷法” 与 “民间法” 并存的 “多元一体” 法 （文化）。二为在现代社会背景之下，将 “官方法”与 “民间法”、“制定法”与 “习惯法”、 “本土法” 与 “移植法” 等多种法律秩序并存、互动、竞争的局面称为 “法律多元”。苏力 指出中国法律的多元存在多个层次：包括外国法律观念、体系与传统法律制度、行为模式、 法律观; 与计划经济相适应的法律制度及与市场经济相适应的法律制度。在其看来 “最根本 的问题是所谓的中国社会传统法律——表现为民间法律——和现代国家法律的冲突问题”。[11] 肖光辉认为 “法律多元意味着法律应该有多种中心、多个层次, 在一定的条件下存在着两个 或两个以上可供人们适用的法律规范, 而不能仅仅只有一个法律规范”。[12]三是也有学者将法 律多元理解为法的概念、功能和渊源的多元性; [13]有的则在社会多元基础上, 将其理解为我 国法律渊源的主要特点。[14]

从上, 可以看出中西方学者对法律多元含义的理解存在诸多差异, 但在笔者看来, 这些 界定的不同，不能简单定位对错或偏颇与否，其归根结底来源于不同学者基于不同学科对同 一主题研究的视角和具体应用场景上的差异。

\section{3 法律多元的研究路径}

学者杨静哲将法律多元的研究路径分为四种：哲学路径、政治理论与传统公法理论的路 径、法学路径、社会科学路径 (以社会学、人类学为代表)。并认为从研究贡献来说, 法学及 社会科学路径是法律多元理论的主导研究路径。

从西方及我国法律多元研究的历史及成果来说，笔者同意杨静哲的分析，法学路径及社 会科学路径的确主导了法律多元理论的研究, 并且尤以社会科学路径为主, 因为在法学研究 框架之内，与国家毫无关联的所谓的 “民间法”、“习惯法” 始终无法纳入规范分析法学的 “法” 概念之中，而这些规则或规范（活法）却又确实地以相似于国家法治理社会的模式发挥着调 整社会秩序的作用。正如格里菲斯强调法律多元概念是社会意义上的而非法律意义上的, 并 认为法律多元应该从社会学的角度来探讨, 而不应该从法、法律或法律系统来研究, 法律多 元与社会多元相伴而生。[16]因而笔者认为 “法律多元” 应是在社会科学及后现代法学讨论框 架内的理论，只有如此，才不会遭遇民间法、习惯法根本不是法的困境。

\section{4. 法律多元研究的意义及困惑}

法律多元理论产生于殖民地统治的背景下，后又辐射于现代社会中国家法不能或不及的 领域。在笔者看来, 法律多元理论研究取得了一定的成就, 但就目前的研究内容及水平看, 仍存困惑。 


\section{1 法律多元研究的意义}

（1）“法律多元” 是法人类学、法社会学的核心精神, 因而该理论的产生和发展打通了 法学与其他社会学科的联系, 学者们开始对传统法学进行反思并对法的现代性进行思考, 此 为法律多元研究的理论意义;

(2) 对于绝大多数以移植西方法律制度为法制现代化主要手段的发展中国家来说, 通过 对法律多元的研究, 学者们考查移植法的实效并思考了 “本土资源” (苏力语) 对于本国法治 建设的意义，此为法律多元研究的实践价值;

（3）法律多元理论质疑国家法一元的地位, 关注非国家法的作用, 因而对中国民间法研 究、法的全球化研究, 兼具理论与实践双重意义。

\section{2 法律多元研究的困惑}

时至今日, 法律多元论仍有一些困惑及不足。有学者认为 “晚近法律多元论繁荣发展的 背后暗藏着三重困境: 即关于法律本身两种属性（指事实之维与规范之维：笔者注）的 “整 合” 问题、关于国家秩序/体系的 “定位” 问题以及关于研究领域的 “划界” 问题（指法律多 元论是法学相关学科的分支还是独立研究领域: 笔者注)”。[17]在笔者看来, 除上述困境, 法 律多元论的研究仍存在以下困惑:

(1) 作为法律多元论主导研究路径且偏重于事实描述与分析的社会学研究方法在对法律 现象的研究上, 是否或者怎样才能做到由个别到一般, 从描述具体事实再落实到规范的制定 与有效实施上（尤其在有成文法传统的中国）? 笔者从法学学者立场出发, 认为此为法律多 元研究中最核心的问题。

（2）对于 “什么是法?” 的解答, 在规范法学的视野中, 法必须由国家产生或承认, 因 而我们所说的 “非国家法” 其实根本不是法, 这一直是法律多元研究中的困境, 正如梅丽的 感慨: “为什么为非国家法寻找一个词是如此之难? ……称所有这些形式的秩序为法律有用 吗? 在关于法律多元的写作中, 我发现一旦法律中心主义被打破, 往往用法律这一术语来指 称那些不属于国家法的秩序形式就会混淆了分析”。[18]而格里菲斯也明确指出, 所谓 “法律多 元” 实际应理解为 “社会调整规范多元”, [19]同时笔者在阅读我国一些学者的习惯法著作时, 经常会有 “书里面记载的民俗习惯是法吗? ” 的疑惑。那么在法律多元的研究范畴内, “法” 与 “非法” 的界限如何划分?

（3）众多中西方学者对 “法律多元” 进行了界定, 其共同点是均认为法律应有多种形式, 但该 “多元” 的场阈到底是限定在某一具体事件、一个社会、一个国家抑或一特定时空内进 行描述?

（4）是否应关注、强调具体的 “二元对立” 法秩序情形? 在法律多元的研究中, 有学者 注意到了社会中存在多种法秩序, 进而引出了法律多元的论断。但也有一些学者不仅注意到 了多种法秩序的存在, 而且强调了具体的二元对立情形 (如千叶正士的三重二分法及我国学 者对国家法一—民间法、本土法——移植法、国内法——国际法等论题的讨论), 那么微观的 “二元对立” 法秩序的研究是否应该是未来法律多元研究的主导趋势?

\section{5. 结语}

萨维尼曾说过, “法律首先产生于习俗和人民的信仰, 其次乃假手于法学一职是之故, 法 律完全是由沉潜于内、默无言声而孜孜矻矻的伟力, 而非法律制定者的专断意志所孕就的”。 [20]罗斯科 - 庞德在其著作《通过法律的社会控制》中把社会控制视为对人类本性的控制, 而 法律仅是道德、宗教等几种社会控制中最重要的一种。 ${ }^{[21]}$ 本文对法律多元的理论要点进行了 展示和剖析, 意在该理论的视阈内, 正视多元社会调整规范存在的事实, 并进一步探讨多元 规范与国家法互动融合的路径, 进而收益于中国的法治建设。 


\section{References}

[1] Zhang Jinfan, The Legal Culture of Diversification and Unity: The Chinese Legal System Which Contains Ethnic Minorities' Legal Wisdom, Ethno-National Studies, pp. 1, 2011(05).

[2] Zhou Xiaohong, Legal Pluralism in the Context of Globalization, ph.D. Dissertation of JiLin University, pp. 4-5, 2006.

[3] B. Tamanaha, What is "General" Jurisprudence? A Critique of Universalistic Claims by philosophical Concepts of Law, 3 Transnational Legal Theory(2012) , available at: http:/ / ssrn. com / abstract $=2018283(2012-9-1)$. Yang Jingzhe. Legal Pluralism: Trajectory, Dilemma and Solution, Science of Law (Journal of Northwest University of Political Science and Law), pp. 8, 2013(2).

[4] Masaji Chiba, Legal Pluralism: Toward a General Theory through Japanese Legal Culture. Qiang Shigong etc., BeiJing: China University of Political Science and Law Press, pp. 1, 1997.

[5] J. Vanderlinden, Return to Legal Pluralism Twenty Years Later, 28 Journal of Legal Pluralism, pp.149-157, 1989.

[6] Sally Engle Merry, Legal Pluralism, Law and Society Review, Vol. 22, Number.5, pp. 870, 1988.

[7] Laura Nader, The Ethnography of Law, American Anthropologist, New Series, Vol. 67, Issue 6, pp. 26, 1965.

[8] Hanne Petersen, Henrik, Zahle, Legal Polycentricity, Aldershot: Darmouth Publishing, pp. 8, 1995.

[9] B. de Sousa Santos, The Law of the Oppressed: The Construction and Reproduction of Legality in Pasargadae, 12 Law \&Society Review, pp. 5-126, 1977. Yang Jingzhe. Legal Pluralism: Trajectory, Dilemma and Solution, Science of Law (Journal of Northwest University of Political Science and Law), pp. 4, 2013(2).

[10]Masaji Chiba, Legal Pluralism: Toward a General Theory through Japanese Legal Culture. Qiang Shigong etc., BeiJing: China University of Political Science and Law Press, pp. 183-193, 1997.

[11] Su Li, Evasion of Law and Legal Pluralism, Peking University Law Journal, pp. 18, 1993(6).

[12]Xiao Guanghui, Legal Pluralism and the Analysis of Issues of Legal Pluralism, Academic Forum, pp. 118, 2007(4).

[13] Shi Tongbiao, Analysis on Stig Jogensen's Pluralistic Jurisprudence, China Legal Science, pp. 102-109, 1993(3).

[14] Ma Yan and Li Yuquan, On the Value Orientation of Legislation in Pluralistic Society, Contemporary Law Review, pp. 15, 2001(3).

[15] Yang Jingzhe. Legal Pluralism: Trajectory, Dilemma and Solution, Science of Law (Journal of Northwest University of Political Science and Law), pp. 3-5, 2013(2).

[16]Griffiths, What Is Legal Pluralism, Journal of Legal Pluralism and unofficial Law,nr.24,1986. Xiao Guanghui, Legal Pluralism and the Analysis of Issues of Legal Pluralism, Academic Forum, pp. 118, 2007(4).

[17] Yang Jingzhe. Legal Pluralism: Trajectory, Dilemma and Solution, Science of Law(Journal of Northwest University of Political Science and Law), pp. 7, 2013(2). 
[18] Sally Engle Merry, Legal Pluralism, Law and Society Review, 1988, Vol. 22, Number5. Hong Han, Legal Pluralism from the Perspective of Legal Anthropology, Journal of Yunnan University Law Edition, Vol. 25, pp. 137, 2012(4).

[19] Griffiths, What Is Legal Pluralism, Journal of Legal Pluralism and unofficial Law, nr.24, pp. 1, pp. 38-39,1996.

[20]F. C. V. Savigny, Of the Vocation of Our Age for Legislation and Jurisprudence. Xu Zhangrun, BeiJing: China Legal Publishing House, pp. 8, 2007.

[21]Roscoe Pound, Social Control through Law: The Task of Law. Shen Zongling and Dong Shizhong, BeiJing: Commercial Press, pp. 33, 1984. 